\title{
Spin-Dependent Electron Scattering at Graphene Edges on Ni(111)
}

\author{
A. Garcia-Lekue, ${ }^{1,2}$ T. Balashov, ${ }^{3}$ M. Olle, ${ }^{3}$ G. Ceballos, ${ }^{3}$ A. Arnau, ${ }^{1,4,5}$ P. Gambardella, ${ }^{3,6,7}$ \\ D. Sanchez-Portal, ${ }^{1,4}$ and A. Mugarza ${ }^{3}$ \\ ${ }^{1}$ Donostia International Physics Center (DIPC), Paseo Manuel de Lardizabal 4, E-20018 San Sebastián, Spain \\ ${ }^{2}$ IKERBASQUE, Basque Foundation for Science, E-48011 Bilbao, Spain \\ ${ }^{3}$ Catalan Institute of Nanoscience and Nanotecnology (ICN2), UAB Campus, E-08193 Bellaterra, Spain \\ ${ }^{4}$ Centro de Física de Materiales CFM - MPC, Centro Mixto CSIC-UPV, Paseo Manuel de Lardizabal 5, E-20018, San Sebastián, Spain \\ ${ }^{5}$ Dpto. de Física de Materiales UPV/EHU, Facultad de Quimica, Paseo Manuel de Lardizabal 3, E-20018, San Sebastián, Spain \\ ${ }^{6}$ Instituciò Catalana de Recerca i Estudis Avancats (ICREA), E-08193 Barcelona, Spain \\ ${ }^{7}$ Department of Materials, ETH Zurich, Hönggerbergring 64, CH-8093 Zurich, Switzerland
}

(Received 12 September 2013; published 14 February 2014)

\begin{abstract}
We investigate the scattering of surface electrons by the edges of graphene islands grown on $\mathrm{Ni}(111)$. By combining local tunneling spectroscopy and $a b$ initio electronic structure calculations we find that the hybridization between graphene and Ni states results in strongly reflecting graphene edges. Quantum interference patterns formed around the islands reveal a spin-dependent scattering of the Shockley bands of $\mathrm{Ni}$, which we attribute to their distinct coupling to bulk states. Moreover, we find a strong dependence of the scattering amplitude on the atomic structure of the edges, depending on the orbital character and energy of the surface states.
\end{abstract}

DOI: 10.1103/PhysRevLett.112.066802

Understanding electron scattering at graphene edges and domain boundaries is fundamental to control transport and quantum confinement in graphene-based electronic devices [1-3]. Edges and boundary defects play an important role in electron transport across multidomain, mesoscopic graphene layers [4,5], as well as in inducing energy gaps $[6,7]$ and magnetic order [8] in graphene nanostructures.

A particularly relevant issue for the performance of nanodevices is the scattering of electrons at the interface between graphene and metal contacts, which determines the charge and spin injection efficiency into graphene. Weakly interacting metal contacts simply dope the Dirac bands $[9,10]$. In such a case, scattering depends only on the energy match between undistorted graphene and metal states. The interface with more reactive metals, however, is usually characterized by significant electronic reconstruction, which defines a complex scenario for scattering. The graphene-Ni interface represents an interesting case where the interaction with the ferromagnetic substrate opens hybridization gaps [11-14] and induces magnetic moments [15]. Consequently, graphene is predicted to behave as a perfect spin filter in contact with a magnetic Ni electrode [13,14], as suggested also by the high spin injection efficiency measured in $\mathrm{Ni}_{80} \mathrm{Fe} 20 /$ graphene/Si heterostructures [16]. Previous studies focused on electron injection perpendicular to the interface, whereas edge scattering in the most common current-in-plane geometry, remains unexplored.

In this Letter, we investigate electron scattering at the edges of graphene on a $\mathrm{Ni}(111)$ substrate. We grow graphene nanoislands with well-defined edge geometries in order to simultaneously probe the electronic structure of
PACS numbers: 73.20.-r, 68.37.Ef, 72.25.Mk, 72.80.Vp

the vertical and lateral graphene interfaces and compare it with that of the pristine Ni surface. We find clear signatures of spin- and edge-dependent electron scattering revealed by local tunneling spectroscopy measurements combined with spin-polarized $a b$ initio electronic structure calculations. This behavior is attributed to the strong distortion of the electronic structure at the interface, where the $\mathrm{Ni}$ surface states significantly shift in energy and space due to the confinement induced by the graphene layer, and to the different coupling to bulk states of majority and minority $\mathrm{Ni}$ states. We further demonstrate that edge scattering is strongly structure dependent, with asymmetries in the reflection amplitude of up to $30 \%$ for reconstructed and nonreconstructed zig-zag edges. These results suggest the possibility of lateral spin filtering for graphene layers, and demonstrate the importance of designing nanostructures with well-defined edges to control electron or spin transport and confinement in graphene.

The experiments were performed using a scanning tunneling microscope (STM) operated at $5 \mathrm{~K}$ in ultrahigh vacuum. The Ni(111) single crystal was cleaned by cycles of $\mathrm{Ar}^{+}$sputtering and annealing to $925 \mathrm{~K}$. Graphene nanoislands were grown from the catalytic decomposition of propene $\left(\mathrm{C}_{3} \mathrm{H}_{6}\right)$ on a clean $\mathrm{Ni}(111)$ single crystal. Islands with straight edges and either triangular or hexagonal shape were obtained by controlling the reaction temperature and annealing conditions, following the method presented in Ref. [17]. Spectroscopic measurements were performed by STM using the lock-in technique, with a bias voltage modulation of frequency $3 \mathrm{kHz}$ and amplitude $1 \mathrm{mV}_{\text {rms }}$ for the $d I / d V$ spectra, and of $15 \mathrm{mV}_{\text {rms }}$ for the $d I / d V$ maps. The $a b$ initio calculations of the electronic structure were 
(a)
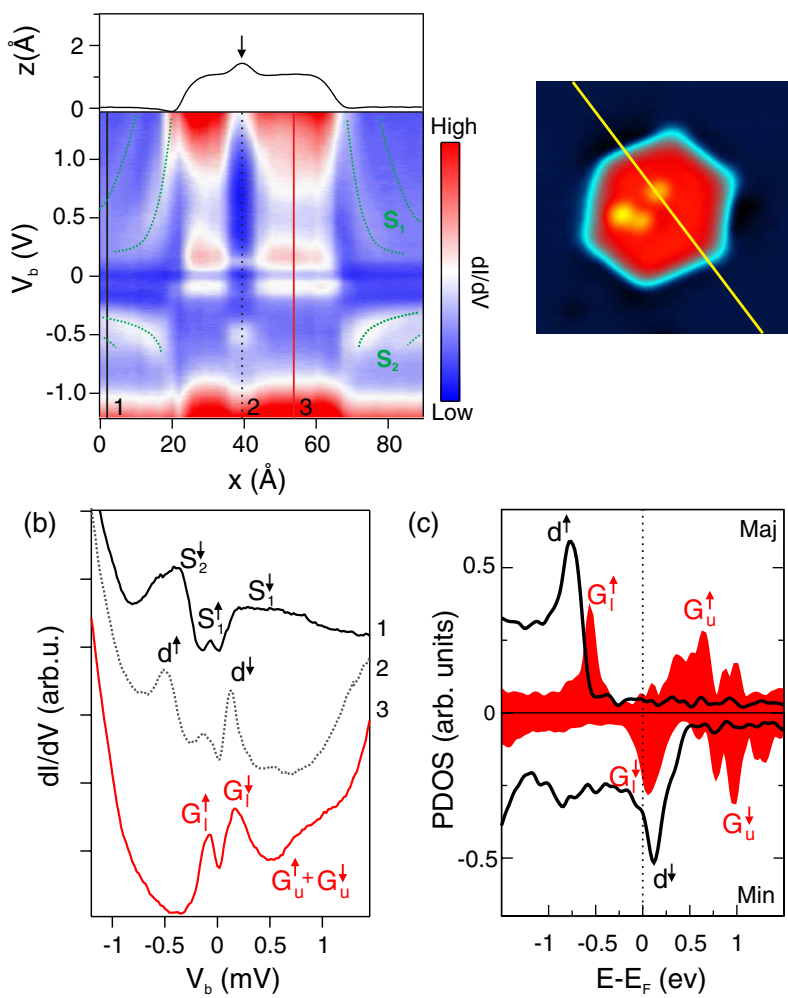

FIG. 1 (color online). (a) Topographic profile and constant height $d I / d V$ spectra taken along the yellow line that crosses the hexagonal island in the STM image shown on the right. The standing waves arising from scattering of the $S_{1}$ and $S_{2}$ surface states are indicated by green lines. Setpoint values: $I=4 \mathrm{nA}$, $V_{b}=-3.0 \mathrm{~V}$. (b) $d I / d V$ spectra of the Ni surface (black), graphene island (red), and of a Ni impurity in the island (dotted grey). (c) Calculated density of states of majority and minority states, projected onto $\mathrm{C}$ (red areas) and $\mathrm{Ni}$ (black line) atoms. Graphene bands are labeled as $G_{u / l}^{\uparrow / \downarrow}$ for spin up/down $(\uparrow / \downarrow)$ and upper and lower band $(u / l)$. For visualization purposes, the PDOS of Ni has been divided by 50 .

carried out using density functional theory, as implemented in the SIESTA code [18]. We use a supercell description of the system, consisting of a slab containing 13 layers of $\mathrm{Ni}(111)$, covered by a single graphene layer on each side. For pristine Ni(111), we employ a 19-layer slab in order to avoid interactions between surface states on the two opposite sides. Further details on the calculations are given in the Supplemental Material [19].

We investigate first the local electronic structure of the graphene islands and surrounding Ni surface, focusing on how the electronic states of both graphene and $\mathrm{Ni}$ are mutually perturbed at the interface. Figure 1(a) shows a series of $d I / d V$ spectra taken along a line that crosses a hexagonal graphene island and contains one impurity [arrow in Fig. 1(a)]. The impurity consists of one or more $\mathrm{Ni}$ atoms that get trapped during the formation of the graphene islands [17]. Representative $d I / d V$ spectra of the Ni surface, Ni impurity, and graphene nanoisland are shown in Fig. 1(b). The spectrum acquired on graphene (a)

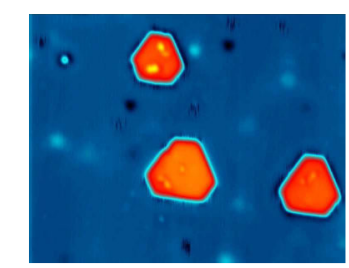

(c)

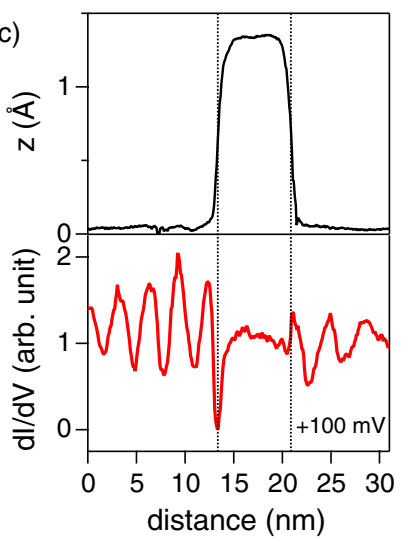

(b)

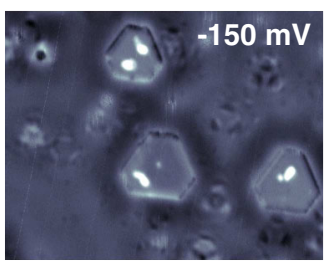

FIG. 2 (color online). (a) Topographic $\left(V_{b}=0.1 \mathrm{~V}\right)$ and (b) constant current $d I / d V$ maps simultaneously acquired at different energies, showing the interference patterns of the $S_{1}$ surface state scattered from graphene islands. Setpoint current: $I=0.3 \mathrm{nA}$. Image size: $30 \times 37 \mathrm{~nm}^{2}$. (c) Topographic and $d I / d V$ profiles along the yellow line in (b), illustrating the absence of interference patterns inside the island.

shows two prominent peaks around $\mathrm{E}_{F}$ followed by a weaker and broad hump centered at about $+0.80 \mathrm{eV}$. First principles calculations assign them to graphene $\pi$ states [Fig. 1(c)], which split into spin-polarized gapped bands $G_{u / l}^{\uparrow \uparrow \downarrow}$ due to the strong hybridization with Ni $d$ bands $[13,14,19-21]$. The spin-split Ni $d$ bands can be clearly identified as the two sharp peaks $d^{\uparrow}$ and $d^{\downarrow}$ in the impurity spectrum; on the Ni surface such peaks are masked by the dominant contribution of surface states, which we label as $S_{1}$ and $S_{2}$ following the nomenclature of previous studies [22-24] (further information on the electronic structure of the surface states can be found in Fig. S2 of the Supplemental Material [19]).

In the following we will examine how the Ni surface states are affected by the interaction with graphene, and how this interaction is determinant for scattering at the graphene edge. The strong spatial variations of the $d I / d V$ intensity outside the graphene island [green lines in Fig. 1(a)] are due to the quantuminterference between incident and reflecting surface electrons. The interference patterns formed by $S_{1}$ at different energies are clearly visible in the $d I / d V$ maps and profile shown in Fig. 2. This shows that the Ni surface states are very sensitive to the presence of graphene edges. Moreover, contrary to graphene nanoislands grown on $\operatorname{Ir}(111)[25,26]$, the wave patterns are absent inside the islands. Our ab initio calculations attribute this effect to the significant modification of the $S_{1}$ surface state below the graphene layer, which 


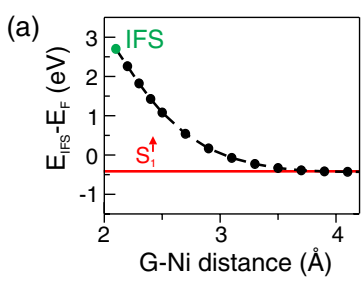

(b)

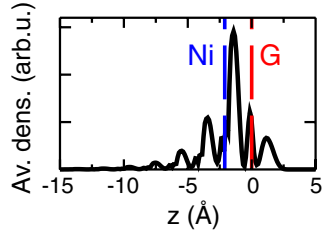

(c)

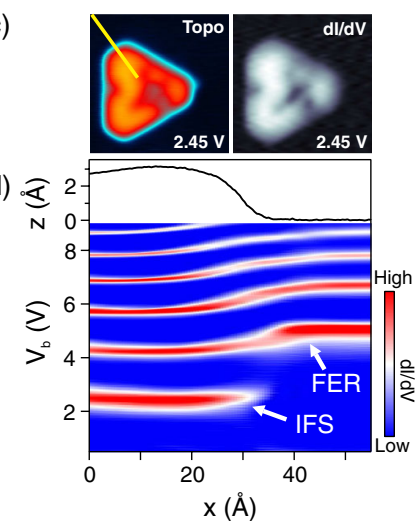

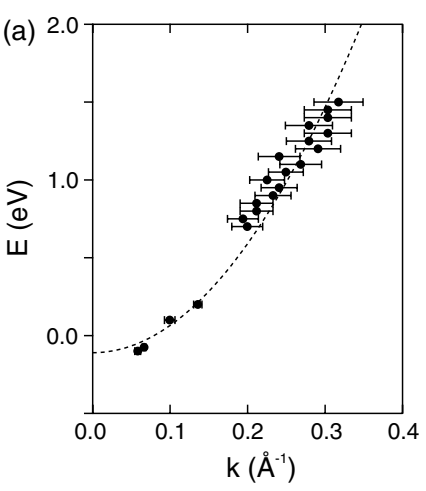

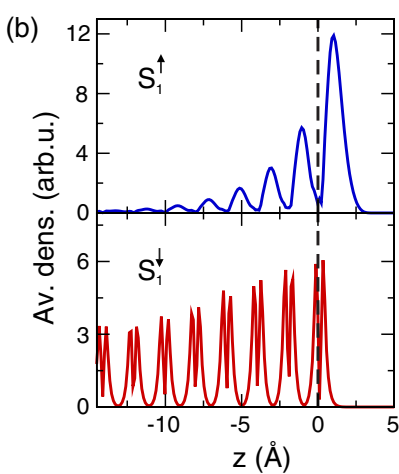

FIG. 4 (color online). (a) Dispersion relation obtained from the standing wave periodicity. A parabolic curve is included as a guide (dashed line), with onset at $-0.110 \mathrm{eV}$, as obtained from the $d I / d V$ spectra. (b) Planar average density of the majority and minority $S_{1}$ states at the $\Gamma$ point.

in Fig. 2(b) [29]. We find a single dispersion curve [Fig. 4(a)] with no evidence for exchange-split bands. This curve is assigned to the majority $S_{1}^{\uparrow}$ sate, in agreement with all the band structure calculations reported to date, which predict $S_{1}^{\uparrow}$ to be partially occupied in clear contrast to the minority $S_{1}^{\downarrow}[19,22,23,30,31]$. Another strong argument for such assignation is provided by the different surface character of majority and minority $S_{1}$ bands, as depicted in Fig. 4(b). The minority band, lying much closer to bulk states [19], presents a substantially shorter lifetime and a larger penetration into the bulk, illustrated in Fig. 4(b) by the density profile of each of those bands at $\Gamma$. At this point, $S_{1}^{\downarrow}$ overlaps with bulk bands and assumes a surface resonance character. Scattering to bulk states at the graphene edge further reduces the lifetime of $S_{1}^{\downarrow}$ electrons. This explains the dominant contribution of the majority states to the standing waves and supports the identification of the experimental curve in Fig. 4(a) with the dispersion of the $S_{1}^{t}$ band. The overall effect is a spin-dependent scattering that is mainly due to the different absorption to bulk states at the graphene edges, as opposed to the spin-dependent transmission in the scattering of Ni d states perpendicular to the interface $[13,14]$.

Finally, we investigate the influence of the edge geometry on electron scattering. Hexagonal islands are ideal for this purpose, since edges on adjacent sides present a distinct atomic structure, as shown in Fig. 5(a). The two different edge types correspond to an unreconstructed zig-zag edge $(n)$ and a reconstructed edge $(r)$ with double periodicity that is related to the so-called " 57 " reconstruction [32], in which the outermost hexagons are replaced by pentagons and heptagons [2]. The asymmetry in the scattering amplitude at two opposite edges is evident in Figs. 5(b) and (c). From the spectroscopic images shown in (c) we observe that reconstructed edges produce weaker $d I / d V$ oscillations at most energies. At $+1.6 \mathrm{~V}$, intensity modulations also appear at the graphene side of the edge, which could either 
(a)

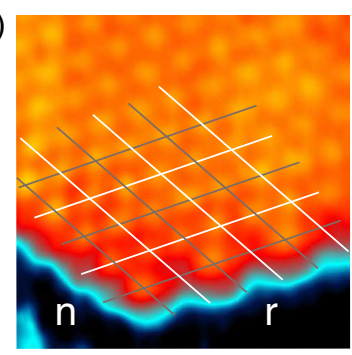

(c)

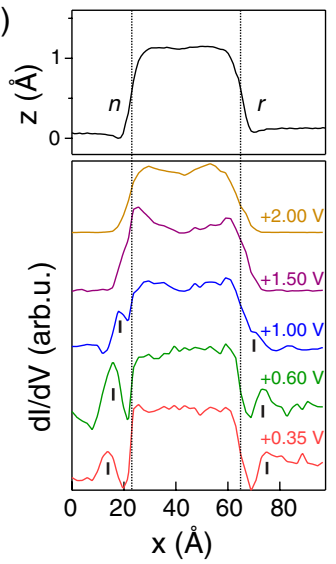

(b)

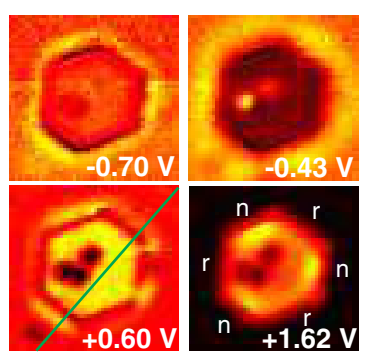

(d)

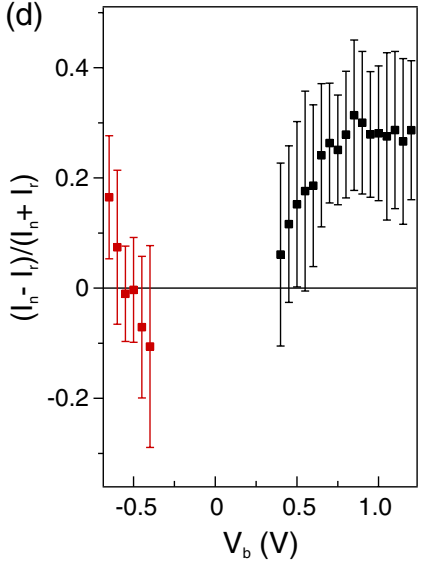

FIG. 5 (color online). (a) Atomically resolved topographic image of the boundary of a graphene island, where reconstructed edges $(r)$ exhibit twice the periodicity of the nonreconstructed ones (n). (b) Constant height $d I / d V$ maps measured on a $50 \times 50$ grid on the hexagonal island of Fig. 1. Setpoint values: $I=4 \mathrm{nA}, V_{b}=-3.0 \mathrm{~V}$. (c) Topographic and $d I / d V$ profiles taken along the green line in (b). For better visualization, the profiles are normalized by subtracting the $d I / d V$ intensity of the $\mathrm{Ni}$ surface and divided by the intensity at the center of the island. (d) Asymmetry of the scattering intensity of $n / r$ edges for the $S_{1}$ (black) and $S_{2}$ (red) surface state, as defined in the text. Error bars indicate the standard deviation of data obtained from different edges of the hexagon.

indicate asymmetric scattering of the graphene bands in this energy range (see Fig. S3 in Ref. [19]) or the presence of edge states. The asymmetry effect can be better quantified by measuring the $d I / d V$ intensity $\left(I_{n, r}\right)$ at the first maximum of the standing wave as a function of energy, indicated by ticks in Fig. 5(b). Both edges are excellent reflectors for the $S_{1}$ states due to the large energy shift of this band in the graphene covered region, which effectively inhibits transmission. Therefore, the peak intensity ratio for the two edge types is mainly determined by the differences in the reflection/absorption ratio [33]. Absorption here means both elastic and inelastic scattering into bulk states. Figure 5(d) shows the edge scattering asymmetry defined as the ratio $\left(I_{n}-I_{r}\right) /\left(I_{n}+I_{r}\right)$. Positive values $\left(I_{n}>I_{r}\right)$ thus imply larger absorption at reconstructed edges . We see that, for $S_{1}$, the asymmetry increases as we go higher in energy (away from the $\Gamma$ point), saturating at a value of about $30 \%$ at $+0.8 \mathrm{~V}$. The increment of the asymmetry is consistent with the periodicity doubling of the $r$ edge that, due to band

folding, is likely to increase the absorption into bulk. On the other hand, the downwards dispersing $S_{2}$ band shows a more complex behavior, with a negative asymmetry at $\Gamma$ that changes sign at lower energy. Therefore, the edge type, electron energy, and orbital composition of the surface state concur in determining the scattering asymmetry.

In conclusion, graphene nanoislands with well-defined edge geometry grown on $\mathrm{Ni}(111)$ allowed us to study the scattering of two-dimensional electrons parallel to the graphene-metal interface. The strong interaction between $\mathrm{C}$ and $\mathrm{Ni}$ atoms induces a significant energy mismatch of the surface bands inside and outside graphene, quenching the transmission through the graphene edge. In the case of the $S_{1}$ surface state of $\mathrm{Ni}$, this effect is modulated by the different degree of coupling to bulk states of each spin-split band, leading to pronounced spin-dependent scattering that favors the reflection of majority electrons. The atomic edge structure has a significant influence on the scattering amplitude, leading to a scattering asymmetry for the majority $S_{1}$ band of up to $30 \%$. These results elucidate the complex scattering properties of graphene-metal interfaces and are important for the control of electron transport and quantum confinement in lateral graphene junctions with spin-polarized electrodes.

We acknowledge support from the Basque Departamento de Educación, UPV/EHU (Grants No. IT-366-07 and No. IT756-13), the Spanish Ministerio de Ciencia e Innovación (Grants No. FIS2010-19609-C02-00, and No. MAT201015659), the ETORTEK program funded by the Basque Departamento de Industria, the European Research Council (StG 203239 NOMAD), and Agència de Gestió d'Ajuts Universitaris i de Recerca (2009 SGR 695). A. M. acknowledges funding from the Ramón y Cajal Fellowship program.

[1] A. H. C. Neto, F. Guinea, N. M. R. Peres, K. S. Novoselov, and A. K. Geim, Rev. Mod. Phys. 81, 109 (2009).

[2] A. Rozhkov, G. Giavaras, Y. P. Bliokh, V. Freilikher, and F. Nori, Phys. Rep. 503, 77 (2011).

[3] M. H. Rümmeli, C. G. Rocha, F. Ortmann, I. Ibrahim, H. Sevincli, F. Börrnert, J. Kunstmann, A. Bachmatiuk, M. Pötschke, M. Shiraishi, M. Meyyappan, B. Büchner, S. Roche, and G. Cuniberti, Adv. Mater. 23, 4471 (2011).

[4] P. Y. Huang, C. S. Ruiz-Vargas, A. M. van der Zande, W. S. Whitney, M. P. Levendorf, J. W. Kevek, S. Garg, J. S. Alden, C. J. Hustedt, Y. Zhu, J. Park, P. L. McEuen, and D. A. Muller, Nature (London) 469, 389 (2011).

[5] A. W. Tsen, L. Brown, M. P. Levendorf, F. Ghahari, P. Y. Huang, R. W. Havener, C. S. Ruiz-Vargas, D. A. Muller, P. Kim, and J. Park, Science 336, 1143 (2012).

[6] T. Shimizu, D. C. Haruyama, J. Marcano, D. V. Kosinkin, J. M. Tour, K. Hirose, and K. Suenaga, Nat. Nanotechnol. 6, 45 (2011).

[7] S. Roche, Nat. Nanotechnol. 6, 8 (2011).

[8] J. Fernández-Rossier and J. J. Palacios, Phys. Rev. Lett. 99, 177204 (2007). 
[9] G. Giovannetti, P. A. Khomyakov, G. Brocks, V. M. Karpan, J. van den Brink, and P. J. Kelly, Phys. Rev. Lett. 101, 026803 (2008).

[10] P. Sutter, J. T. Sadowski, and E. Sutter, Phys. Rev. B 80, 245411 (2009).

[11] P. Sutter, M. S. Hybertsen, J. T. Sadowski, and E. Sutter, Nano Lett. 9, 2654 (2009).

[12] A. Varykhalov, J. Sánchez-Barriga, A. M. Shikin, C. Biswas, E. Vescovo, A. Rybkin, D. Marchenko, and O. Rader, Phys. Rev. Lett. 101, 157601 (2008).

[13] V. M. Karpan, G. Giovannetti, P. A. Khomyakov, M. Talanana, A. A. Starikov, M. Zwierzycki, J. van den Brink, G. Brocks, and P. J. Kelly, Phys. Rev. Lett. 99, 176602 (2007).

[14] J. Maassen, W. Ji, and H. Guo, Nano Lett. 11, 151 (2011).

[15] M. Weser, Y. Rehder, K. Horn, M. Sicot, M. Fonin, A. B. Preobrajenski, E. N. Voloshina, E. Goering, and Y. S. Dedkov, Appl. Phys. Lett. 96, 012504 (2010).

[16] O. M. J. van 't Erve, A. L. Friedman, E. Cobas, C. H. Li, J. T. Robinson, and B. T. Jonker, Nat. Nanotechnol. 7, 737 (2012).

[17] M. Olle, G. Ceballos, D. Serrate, and P. Gambardella, Nano Lett. 12, 4431 (2012).

[18] J. M. Soler, E. Artacho, J. D. Gale, A. Garcia, J. Junquera, P. Ordejón, and D. Sanchez-Portal, J. Phys. Condens. Matter 14, 2745 (2002).

[19] See Supplemental Material at http://link.aps.org/ supplemental/10.1103/PhysRevLett.112.066802 for detailed explanation of the $a b$ initio calculations, and the effect of $\mathrm{Ni}$ impurities on the electronic properties of graphene.

[20] M. Weser, E. N. Voloshina, K. Horn, and Y. S. Dedkov, Phys. Chem. Chem. Phys. 13, 7534 (2011).

[21] L. V. Dzemiantsova, M. Karolak, F. Lofink, A. Kubetzka, B. Sachs, K. von Bergmann, S. Hankemeier, T. O. Wehling, R. Frömter, H. P. Oepen, A. I. Lichtenstein, and R. Wiesendanger, Phys. Rev. B 84, 205431 (2011).
[22] J. Lobo-Checa, T. Okuda, M. Hengsberger, L. Patthey, T. Greber, P. Blaha, and J. Osterwalder, Phys. Rev. B 77, 075415 (2008).

[23] T. Ohwaki, D. Wortmann, H. Ishida, S. Blügel, and K. Terakura, Phys. Rev. B 73, 235424 (2006).

[24] Y. Nishimura, M. Kakeya, M. Higashiguchi, A. Kimura, M. Taniguchi, H. Narita, Y. Cui, M. Nakatake, K. Shimada, and H. Namatame, Phys. Rev. B 79, 245402 (2009).

[25] S. J. Altenburg, J. Kröger, T. O. Wehling, B. Sachs, A. I. Lichtenstein, and R. Berndt, Phys. Rev. Lett. 108, 206805 (2012).

[26] D. Subramaniam, F. Libisch, Y. Li, C. Pauly, V. Geringer, R. Reiter, T. Mashoff, M. Liebmann, J. Burgdörfer, C. Busse, T. Michely, R. Mazzarello, M. Pratzer, and M. Morgenstern, Phys. Rev. Lett. 108, 046801 (2012).

[27] B. Borca, S. Barja, M. Garnica, D. Sánchez-Portal, V. M. Silkin, E. V. Chulkov, C. F. Hermanns, J. J. Hinarejos, A. L. Vázquez de Parga, A. Arnau, P. M. Echenique, and R. Miranda, Phys. Rev. Lett. 105, 036804 (2010).

[28] P. A. Khomyakov, G. Giovannetti, P. C. Rusu, G. Brocks, J. van den Brink, and P. J. Kelly, Phys. Rev. B 79, 195425 (2009).

[29] L. Simon, C. Bena, F. Vonau, M. Cranney, and D. Aubel, J. Phys. D 44, 464010 (2011).

[30] J. Braun and M. Donath, Europhys. Lett. 59, 592 (2002).

[31] G. Bertoni, L. Calmels, A. Altibelli, and V. Serin, Phys. Rev. B 71, 075402 (2005).

[32] M. Olle, A. Garcia-Lekue, D. Sanchez-Portal, A. Mugarza, G. Ceballos, and P. Gambardella (unpublished).

[33] The position of the first maximum with respect to graphene edges, measured at $+0.35 \mathrm{~V}$ and $+0.60 \mathrm{~V}$, only differs by $7 \%-15 \%$ between the two types of edges, indicating minor variations in the phase shift, and hence confirming the negligible role of transmission. 